\title{
Dust Cleaner System for PV Panel using IoT
}

\author{
Digambar Jadhav \\ Assistant Professor \\ DIT Pimpri \\ Pune
}

\author{
Vaibhav Muddebhalkar \\ Assistant Professor \\ DIT Pimpri \\ Pune
}

\author{
Ashok Korke \\ Assistant Professor \\ DIT Pimpri \\ Pune
}

\begin{abstract}
Sun power is a standout amongst the most productive yet clean wellsprings of vitality we approach. There are no expanded fuel expenses or conditions, no connections to toxins, and it's both dependable and reasonable. Obviously, keeping in mind the end goal to bridle sun oriented power you require access to particular innovation. This tech depends on either little scale sun oriented photovoltaic (PV) systems but in main problem of photovoltaic (PV) system soil and dust particles accumulating on photovoltaic (PV) panels reduce the solar energy getting the cells, thereby falling overall power performance. We are solving the problem of this cleaning the PV panels is a problem of great practical engineering interest in solar PV power generation cleaning the photovoltaic (PV) panels is a problem of great practical interest in solar PV power creation. We are solve this problem discuss the methods for dust removal system using the Internet of things IoT.We are developing the simple and useful dust cleaning device and developed novel architecture of dust cleaning system for PV panel using IoT . the main motive for this system is developed system for dust cleaning for PV system using IoT and maintaining the clean PV panel efficiency.
\end{abstract}

\section{Keywords}

Interne of Things, Device Management, Sensor,PV,Dust .

\section{INTRODUCTION}

Over the world step by step expanding the populaces, and expanded use of electric vehicles, electric gadgets, industrial facilities, , the expansion of electric power usage is sure. By and by, the power business is going towards an increasingly Ecological welcoming technique for producing power. The most commonplace sorts of eco vitality plants are $\mathrm{PV}$ (photovoltaic) vitality plants, miniaturized scale hydro control plants and wind turbine control plants or tide control plants. Smaller scale hydro vitality plants rely upon conduit streams - falling separation, volume rate, stream rate, wind vitality control plants rely upon airstream speed, period and rate of repeat of winds, and tide plants rely upon the tides. The inverse of the condition dependent power age strategies notice prior sun based vitality control plants require light, a condition that can be fulfilled in many use cases. Sun oriented plants can be worked on-lattice, off-framework, with or without putting away the created vitality and can serve applications that are kept running amid the day (manufacturing plants) or they can simply repay the utilization of expansive vitality shoppers, decreasing expenses with power. They are broadly utilized for home apparatuses or as power production stations for energetic power systems [1].
The Internet of Things (IoT) is an ongoing correspondence worldview that imagines a not so distant future, in which the objects of regular daily existence will be outfitted with microcontrollers, handsets for computerized correspondence, and reasonable convention stacks that will make them ready to speak with each other and with the clients, turning into an indispensable piece of the Internet [1]. The IoT idea, thus, goes for making the Internet significantly increasingly vivid and inescapable. Besides, by empowering simple access and cooperation with a wide assortment of gadgets, for example, for example, home machines, observation cameras, checking sensors, actuators, presentations, vehicles, etc, the IoT will encourage the improvement of various applications that make utilization of the conceivably tremendous sum and assortment of information produced by such articles to give new administrations to subjects, organizations, and open organizations. This worldview without a doubt discovers application in a wide range of spaces, for example, home mechanization, modern robotization, therapeutic guides, portable medicinal services, old help, shrewd vitality the executives and brilliant lattices, car, traffic the board, and numerous others [2]. Web of Things (IOT) idea is being utilized, which for the most part manages detecting, activating, information assembling, putting away and preparing by interfacing physical and virtual gadgets to the Internet.

\section{A. Internet of Things (IoT)}

IoT is an advancing idea with an expanding scope of uses prompting advancement of new innovations and strategies for the enhancement of IOT condition. Here the things are associated and controlled by the Internet..

IoT includes following six elements [2]:

IoT identification: IoT ID is required to coordinate the name and the administrations according to the need.Identification incorporates tending to the IOT objects, which comprises of two things: Object ID and its location. Distinguishing proof strategy isn't exceptional one, for one of a kind ID of the articles address is required.

IoT Sensing: Sensing is required to gather the important data from the system of related articles and to send it back to the server or cloud. Savvy sensors, actuators or wearable detecting gadgets can be the IOT sensors.

IoT Communication: To convey explicit shrewd administrations we have to associate heterogeneous articles together. This element is given by the IOT correspondence innovation within the sight of lossy and boisterous correspondence interfaces, the hubs of IOT can be worked utilizing low power.

IoT Computation: Semantic in the IOT alludes to extraction of learning carefully by various frameworks to give the required administrations.. 
IoT Services: Identity related administrations, data total administrations, community mindful administrations, Universal administrations are the fundamental IOT services.IOT incorporates three primary framework level qualities

\section{RELATED WORK}

Author[1] describes plan for a custom constructed low power autonomous Industrial IoT controller committed for PV board cleaning arrangement. The controller independently from control procedure of the mechanical gadget, is likewise purposeful to associate with web, to be remotely screen and to be equipped for running VPN, ip tables, and other security includes that present gadgets can't execute

Author [3] describes effect of ruining or residue is a noteworthy test in the utilization of photovoltaic (PV).Author portrays the how to self-governing vehicle robot through clean the PV . A PV control station is demonstrated utilizing the Virtual Robot Experimentation Platform and Robot Operating System (ROS) is utilized for the calculations to control the vehicle. Specialized data with respect to mapping, restriction, way arranging, and snag shirking is introduced.

Author [4] begin with the premises that collection of residue on sun powered boards extensively lessen the productivity of the plant, and acquaint a cleaning bot with help in improving the effectiveness of sun oriented boards. The creators demonstrate the trouble in cleaning the sunlight based boards is influencing sun based gasp proprietors. The arrangement portrayed by the creator is straightforward, yet they figure out how to acquire applicable outcomes - demonstrating an expansion of 70 to $130 \%$ of electric power after the cleaning procedure.

The authors[5] examination key duties to the execution effects, cognizance, and alleviation of power hardship in view of buildup on a Solar Panel. Electrical characteristics of PV (Voltage and flow) are discussed with respect to shading due to dirtying. Shading in light of dirtying is isolated in two classes, explicitly, fragile shading, for instance, air defilement, and hard shading which happens when a solid, for instance, accumulated buildup blocks the daylight. The outcome demonstrates that delicate shade influences the current given by the PV module, yet the voltage continues as before. In hard shading, the execution of the PV module depends on whether a few cells are shaded or all cells of the PV module are shaded. In the event that a few cells are shaded, at that point as long as the unshaded cells get sun based irradiance, there will be some yield despite the fact that there will be a decline in the voltage yield of the PV module.

Author [6] survey of existing examination into the effect of residue testimony on the execution of PV board, especially $\mathrm{PV}$ and furthermore recognizes difficulties to additionally look into around there. The survey on the status of research has been talked about in two stages, Phase-I evaluating the examination from the 1960s till the 1990; Phase-II assessing the post 1990s research around there. In view of the thorough news coverage contemplate a proposal table has been created to direct in the recognizing fitting cleaning/upkeep cycle for PV frameworks in light of the predominant climatic and ecological conditions..

\section{NOVEL LAYERED ARCHITECTURE}

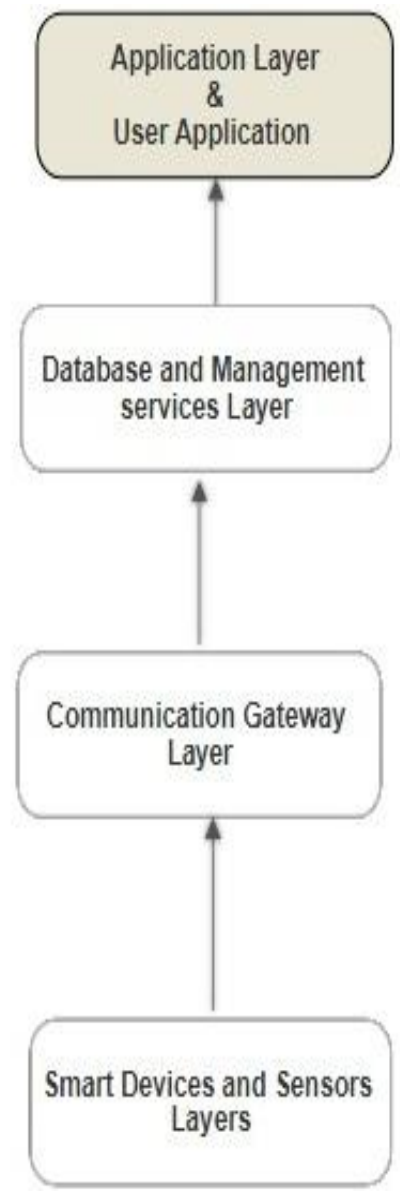

Fig 1: Novel Layered Architecture For Dust Cleaner using IoT

are four layers in fig1. Layer 1. Smart device and sensors layer, Layer 2. Communication Gateway Layer, layer3. Database and Management Service Layer, Layer 4. Application layer and User Application

Layer 1: Smart Device and Sensors Layers

This layer is very important in IoT System.In this layers attach the smart devices such as sensors, RFID etc.this layer fetching raw data from ground layer.

Layer2: Communication Gateway Layer:

This layer using communication medium in IoT system. In this layer used Wifi, Wired, Bluetooth, Zigbee etc. communication gateway .This layer communication medium between Sensor layer and Database layer

\section{Layer3: Database and Management layer}

This Layer processing on raw data and send to application layer also this layer gather data from sensor layer and processing data and send to Application Layer.This layer is very important in IoT because this layer storing data ,processing data these service provide to application Layer.

\section{Layer 4: Application layer/User Application}

This layer provides the suitable result for those who are handled or using IoT application. 


\section{NOVEL ARCHITECTURE FOR DUST CLEANER USING IoT}

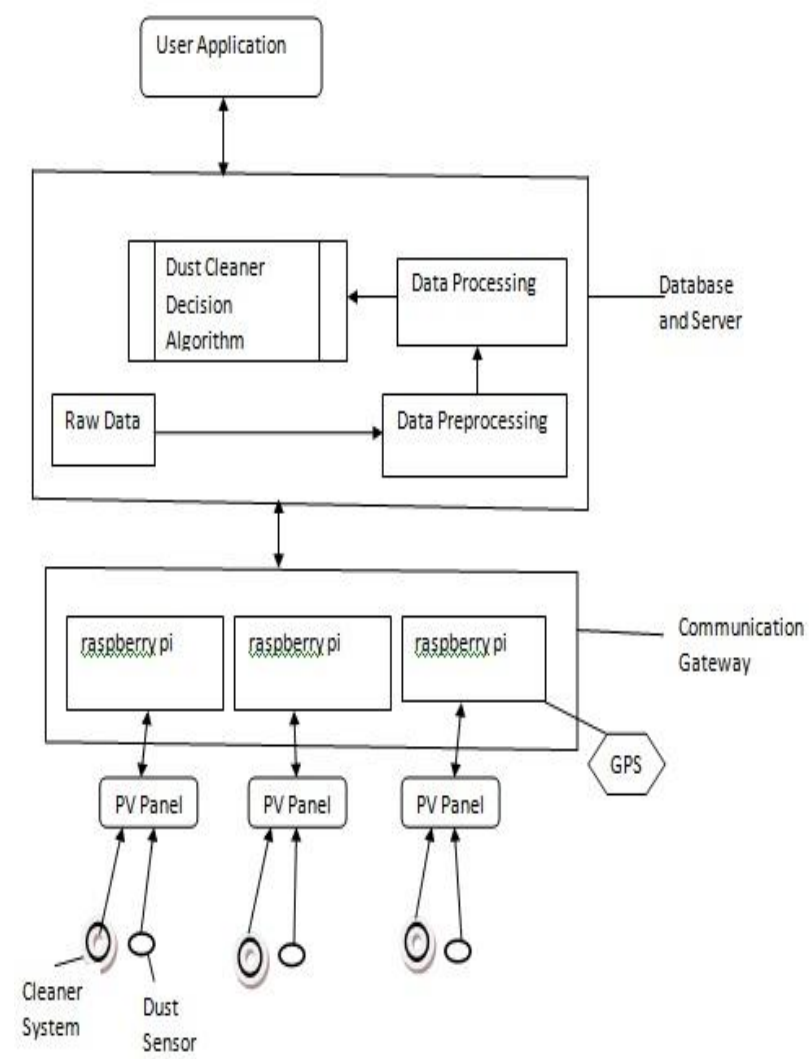

Fig 2: Novel Architecture for Dust Cleaner using IoT

Fig2. Shows PV panel fit into Ground level in Environment each PV panel attach to Dust sensor and Cleaner system .These smart devices connect with Communication gateway (Raspberry pi). The communication gateway collect raw data from dust sensor and send to the data base and server system in Database/server system raw data send to the data preprocessing in data preprocessing transforming raw data into an understandable format and that actual data send to the dust cleaner decision algorithm in this algorithm check the actual data comparing the threshold value of dust if dust data value greater than threshold value the system send to notification to user/admin. Then user send to commend directly dust cleaner and dust cleaner clean the PV panel then again check algorithm value of dust if dust value below threshold value the automatically off the dust cleaner system.

\section{ALGORITHM}

U-User;

DS-Dust Sensor;

TV-Threshold value;

$\mathrm{N}=$ No.of PV Cell;

L-Location;

A-Alart Msg;

C-Cleaner system;

D-Dust Value;

Input:D,TV,N \& L

Output:C
Step1: Start

Step2:DS Start

Step3:if >=TV Then

Find(L);

Find(N);

Alart Msg toU

End

Step4:if A="Yes" \&\& D>=TV Then

U $\operatorname{Start}(\mathrm{C})$;

Else

Off(C);

end

6. ADVANTAGES

1. Biggest benefit of this system is improving light efficiency of PV panel.

2. Automated Dust Clean

3. User Friendly

\section{CONCLUSION}

This work presents a general approach for deploying autonomous cleaner for PV Panel, which can be used for cleaning solar panels. Additional ROS libraries are used for path planning and obstacle avoidance, where the vehicle is able to navigate around the PV power station. Future work will focus on attaching a robotic arm to the vehicle along with the actual simulation of removing dust particles from the panels.

\section{REFERENCES}

[1] Dumitru-Cristian et. al., "Autonomous flexible low power Industrial IoT controller for solar panels cleaning systems ." 21st International Conference on Control Systems and Computer Science. Year -2017 ,Pages 106112

[2] Pallavi K N et. al., "Smart Waste Management using Internet of Things: A Survey." Australian telecommunications networks and applications conference (ATNAC) (2007):175-180.

[3] Nasir K. Memon et.al. "Autonomous Vehicles for Cleaning Solar Panels" Renewable and Sustainable Energy Conference (IRSEC), 2016 International Nov 2016

[4] J. B. Jawale, V. K. Karra, B. P. Patil, P. Singh, S. Singh and S. Atre, "Solar panel cleaning bot for enhancement of efficiency - An innovative approach," 2016 3rd International Conference on Devices, Circuits and Systems (ICDCS), Coimbatore, 2016, pp. 103-108

[5] Mohammad Reza Maghami et.al." Power loss due to soiling on solar panel: A review" Renewable and Sustainable Energy Reviews Volume 59, June 2016, Pages 1307-1316

[6] Monto Mani, Rohit Pillai et.al.'Impact of dust on solar photovoltaic (PV) performance: Research status,challenges and recommendations" Renewable and Sustainable Energy Reviews Volume 14, Issue 9, December 2010, Pages 3124-3131 
[7] Jadhav, Digambar, and Swati Nikam. "Need for Resource Management in IoT." International Journal of Computer Applications 134.16 (2016).

[8] Nikam, Swati, and Digambar Jadhav. "Resource management new system architecture." 2016 International Conference on Computing, Communication and Automation (ICCCA). IEEE, 2016.
[9] Jadhav, Digambar, Vaibhav Muddebhalkar, and Laxman Khandare. "Utilization of Resource's in IoT." International Journal of Computer Applications 167.3 (2017).

[10] Jadhav, Digambar, et al. "Missing Person Detection System in IoT." 2017 International Conference on Computing, Communication, Control and Automation (ICCUBEA). IEEE, 2017. 